\title{
Continuum and all-atom description of the energetics of graphene nanocones
}

\author{
Antonio Šiber \\ Department of Theoretical Physics, Jožef Stefan Institute, SI-1000 Ljubljana, \\ Slovenia \\ Institute of Physics, P.O. Box 304, 10001 Zagreb, Croatia \\ E-mail: asiber@ifs.hr
}

\begin{abstract}
Energies of graphene nanocones with 1 to 5 pentagonal disclinations are studied on an atomically detailed level. The numerical results are interpreted in terms of three different contributions to the cone energy: the core disclination energy, the bending energy of the cone surface, and the "line tension" energy of the cone edge that is related to different coordination of carbon atoms situated at the edge. This continuum description allows for a construction of analytic expressions for the cone energetics and indicates different regimes of cone sizes in which cones with a particular number of disclinations are preferred energywise. An important result of the study is that the energetics of various types of cones profoundly depends upon whether the dangling carbon bonds at the cone basis are saturated by hydrogen atoms or not. This may be of use for explaining the differences in the yields of various cone types in different production processes.
\end{abstract}

PACS numbers: $62.25 .+\mathrm{g}, 61.48 .+\mathrm{c}, 46.25 .-\mathrm{y}$ 


\section{Introduction}

Carbon (C) atoms are famous for their ability to form various hollow structures[1], the best known of them being fullerenes[2] and carbon nanotubes[3]. Soon after the clear experimental detection of carbon nanotubes [3], conical carbon structures have been observed in the process of quenching hot carbon vapour on the graphite substrate [4, 5]. These cones all had the same opening angle, the smallest one of the five that can be constructed from the honeycomb graphene structure (see below). It was later demonstrated that the structures of the same type can also be obtained by other experimental procedures, such as pyrolysis of hydrocarbons in plasma torch [6]. In this procedure, five different types of cone geometries were observed. The yields of conical structures in the prepared samples can be quite high $(\sim 20 \%)[6]$. This fact alone indicates the importance of such structures. In addition, conical graphene structures are also predicted to have very specific mechanical [7] and electronic [8] properties, and could possibly be used as nanoscopic electron-field emitters. Interestingly enough, graphite cones were found in natural samples [9] which additionally highlights the need to understand the occurrence of conical shapes made of carbon.

The polyformity that is characteristic of hollow carbon shapes has its parallel in the structures formed by viral proteins. These also form hollow shells (the so called capsids) that protect the viral genetic material (DNA or RNA). Icosahedral viruses have a structure that is the same as the one exhibited by icosahedral fullerenes [10]. The same proteins that form icosahedral shells may, under different conditions, also form tubular structures, similar in structure to carbon nanotubes. There seems to be a parallel for conical structures also - the core capsids of HIV viruses very much resemble (closed) graphene cone structures which was emphasised in Ref. [11]. The investigation of graphene cones may thus have an added value due to the parallelism of structures found in graphene shapes and viral capsids.

Unlike the fullerenes and carbon nanotubes, conical carbon structures are relatively poorly explored. Not much is known about the optimal conditions for their production. A complete understanding of growth of such structures is also lacking, which is not too surprising since the details of growth of the much more investigated fullerenes and carbon nanotubes are still missing [12. Although there have been some proposals that the formation of cones is most likely due to their "designability", i.e. the fact that they are a combinatorially favorable outcome of the growth [13], it is certainly important to first have a good understanding of and reliable estimates for the cone energies. Various contributions to the cone energetics are not completely self-evident, and it is not straightforward, for example, to estimate the importance of the curvature of the cone surface and its contribution to the total energy of the cone.

In this article I shall examine the energetics of (single-walled) graphene cones. These cones contain multiple pentagonal defects. Cones with defective carbon rings whose number of sides is smaller than 5 are not considered in this study as these are shown to be less stable[14]. To understand various factors that influence the energy of 
the cones numerical, all-atom calculations of the cone energetics are performed. The numerical results are interpreted in terms of different energy contributions that are disentangled from the total energy balance. This enabled a construction of reliable analytical expressions for the cone energetics.

Section 2 discusses the details of the cone geometry and describes a particular construction scheme of the cones that is used in this study.

Section 3 contains the details of the numerical calculations and their results.

In Section 4 the numerical data obtained in Sec. 3 is interpreted in a quite general framework that enables a construction of analytic expressions for the cone energies. Four contributions to the cone energy, each of them having a different physical origin, are identified.

Section 5 contains a discussion of the results and some propositions that may relate the results of the article with the experimental data on carbon cones.

\section{Construction of cones}

There are five different classes of cones that can be constructed from the graphene lattice of carbon atoms. These differ in the total number of disclinations (i.e. pentagonal carbon rings) that they contain close to the cone apex, which is directly related to the cone opening angle, $\theta$. The cones can be conceptually constructed by cutting out 60 degree wedges in the graphene plane, thereby creating a "cut-and-fold" cone "origami" that can be mathematically folded onto a three-dimensional pyramid-like object. Except for the cone with a single pentagonal disclination, this involves creasing along the lines connecting the neighboring pentagons. When the pyramid-like object is relaxed so that its energy is minimal, the shape acquires a cone-like appearance. Additional reconnections between the carbon atoms (e.g. between any two atoms that are on the cone edge and that could possibly form pentagons is they are sufficiently close [15]) are not allowed during the relaxation process.

Except for the cone containing a single pentagonal ring close to its apex, all other cones can be constructed in a multitude of ways, depending on the arrangement of the pentagonal disclinations (see e.g. Ref. [16]). These details are irrelevant for this study, as its main aim is to rationalize the cone energetics in the terms that belong to the realm of continuum physics. In each of the cone classes considered in the following, a fixed arrangement of the carbon pentagons is assumed. An isolated pentagon rule [17] was respected by all of the constructions so that the carbon pentagons do not share C-C bonds - each of the pentagons is completely surrounded by hexagons. The parameter that characterizes the cone is its size, i.e. the total number of carbon atoms that it contains. I shall also assume that the distances between the cone apex and its base, measured along the cone surface, are the same irrespective of the point on the base chosen. In other words, this means that the cones considered can be positioned on their base so that the cone apex projects exactly at the center of the base - the cones considered are right cones. 
The five different cut-and-fold pieces of graphene plane are illustrated in Fig. 1. Similarly looking patterns were recently used for the construction of carbon nanotube caps, which necessarily have six pentagonal disclinations [18]. Note that the definition of the cone apex may be somewhat vague. In fact, it may be better to think of these structures as truncated cones or conical frusta. The smaller/top base of the conical frustum is then defined as the region surrounded the carbon pentagons (for graphene cone with a single disclination, the top base is the carbon pentagon itself). In the limit of very large cones, the distinction between the truncated cone and the regular cone becomes irrelevant since the radius of the bottom base of the cone is much larger than the radius of the cone top base.

It is easy to show that the opening angle of the idealized cone $[\theta$, see Fig. 1f)] can be related to the total number of disclinations $(n)$ as

$$
\theta_{n}=2 \arcsin \left(1-\frac{n}{6}\right)
$$

This produces a discrete sequence of possible opening angles, $\theta_{n}=112.89,83.62,60$, 38.94 , and 19.19 degrees, for $n=1, \ldots, 5$. It shall prove to be of use to consider a (planar) graphene disk as a special case of this construction. In this case, $n=0$, and $\theta_{0}=180$ degrees.

\section{Numerical results for the cone energies}

The energetics of carbon-carbon bonding is simulated by using a second-generation reactive empirical bond-order potential by Brenner et al [19] which belongs to the AbellTersoff class of bond-order potentials. This potential is known to properly account for the anisotropy of $\mathrm{C}-\mathrm{C}$ bonding. It also approximately includes the many-body effects. After the mathematical folding of the piece of graphene plane was performed and the broken $\mathrm{C}-\mathrm{C}$ bonds reestablished along the edges of the shape (this part of the procedure also forms the carbon pentagons), the resulting structure is used as an initial guess for the conjugate gradient procedure that optimizes its shape so as to minimize the total energy. The numerical procedure used for this purpose is described in details in Ref. [20]. Similar computational procedure (the construction of initial mathematically folded shapes and subsequent relaxation using the conjugate gradient method in combination with Brenner's potential) has also been used in Ref. [21].

Figure 2 displays the top and side views of the five types of cones that have been relaxed to their minimum energy configuration. The cones chosen for display are relatively small so that the details of the shape, including the pentagons around the cone apex, can be easily discerned.

Figure 3 displays the energies of the cones depending on the number of $\mathrm{C}$ atoms they contain. In these simulations, the radius of the cut-out shape [denoted by $R$ in Fig. [f)] was varied. The energies displayed are in fact the excess energies $(\Delta E)$, i.e. the extra energy that the cones have with respect to the energy that the same number of carbon atoms would have in the infinite graphene plane. Brenner's potential [19] 
a)

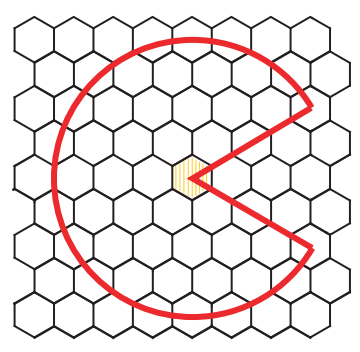

b)

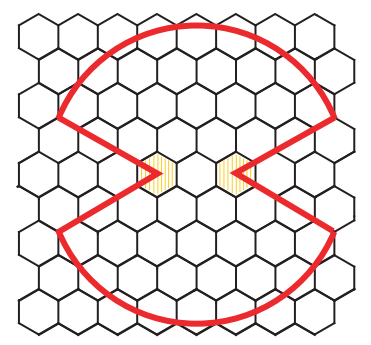

c)

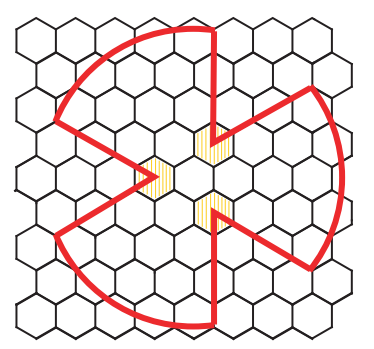

d)

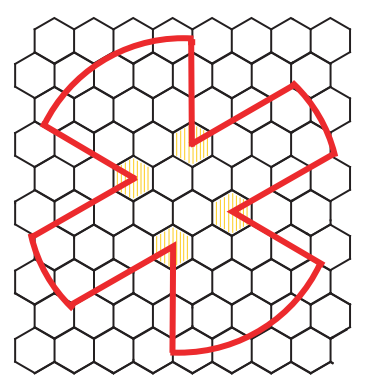

e)

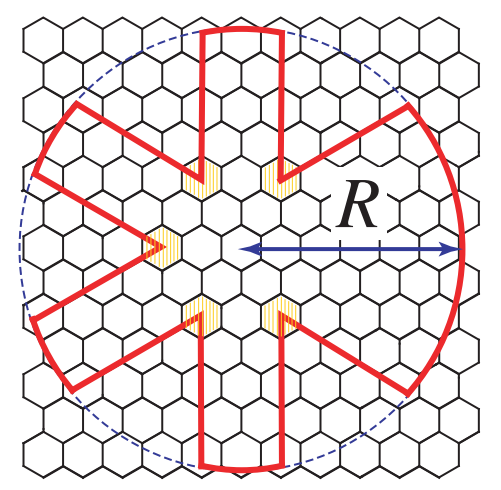

f)

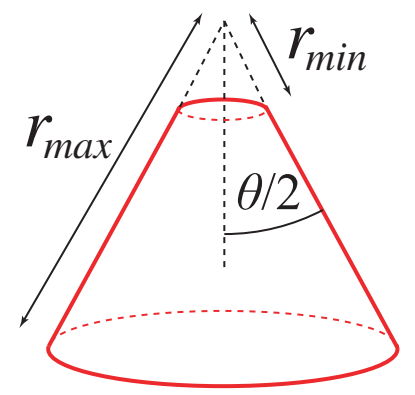

Figure 1. Construction of cones by creation of disclinations (triangular wedges/cuts) in the graphene lattice. The part of the graphene plane bounded by the thick lines is folded into a cone. Panels a), b), c), d), and e) display the graphene cut-outs used to construct the cones with one, two, three, four, and five carbon pentagons, respectively. The size of the cones is determined by the radius $(R)$ of the circle denoted by thin dashed line in panel e). Panel f) represents the cone and the quantities that define it (see text).

predicts that the binding energy per carbon atom is $\epsilon_{b}=-7.39494 \mathrm{eV}$ [21], so that the energy of $I=N \epsilon_{b}$ was subtracted from the total energy of the cones in order to obtain the excess energy. The results for excess energies of graphene disks are also included in Fig. 3. Note the scatter of the data. It is not due to some numerical instability as shall be explained in the next section. 
a)
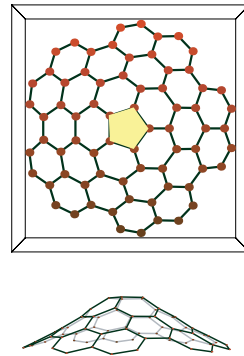

b)

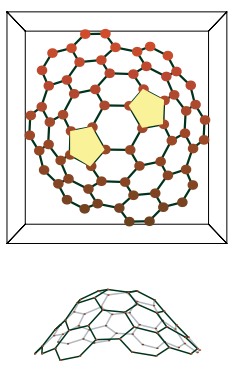

c)

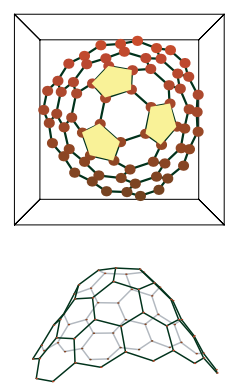

d)
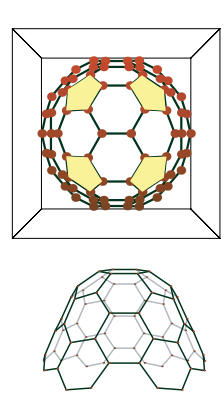

e)

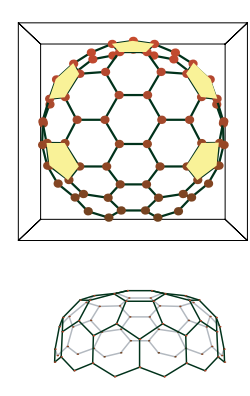

Figure 2. Five different types of cones viewed from above (top row of images) and aside (bottom row of images). Panels a),b),c),d), and e) represent the $n=1, n=2$, $n=3, n=4$, and $n=5$ cones, respectively. The cones contain $70,70,69,72$, and 74 $\mathrm{C}$ atoms for $n=1,2,3,4$, and 5 , respectively.

\section{General considerations of the cone energetics and interpretation of numerical data}

To understand the numerical results obtained in previous section, it helps to isolate four different contributions to the energy of a cone. First, there is a part of the energy that is directly related to the number of diclinations in the cone. Each of the pentagonal disclination (defect) carries the so-called disclination core energy $(D)$ [21, 22] that is related to change (reduction) of the local coordination of entities in the disclination. Second, the cones have a curved surface, so that there is a contribution to the total energy that is of the bending type $(B)$ and directly related to nonvanishing curvature of the cone surface. For $n \neq 0$ this energy can be expressed as [21]

$$
B=\pi c_{0} \frac{\cos ^{2} \theta_{n}}{\sin \theta_{n}} \ln \left(\frac{r_{\max }}{r_{\min }}\right),
$$

where $c_{0}$ is the graphene bending rigidity [21], and $r_{\min }$ and $r_{\max }$ are the distances from the cone apex to the approximate top and bottom bases of the cone, respectively [see Fig. 1f)]. Equation 2 does not include the contribution related to bending of the shape surface in the top base, i.e. in the region where the disclinations accumulate. Assuming that the top base is nearly flat, or that the number of atoms that make it is much smaller from the total number of atoms in a whole conical shape, this part of the energy can be neglected. Third, there is an energy resulting from the coordination of the entities in a shape. This energy can be approximated as a sum of the energy that the same number of carbon atoms would have in the infinite graphene sheet (negative, binding contribution, $I$ ) and the cone-edge energy related to reduced coordination of atoms located along the line that defines the bottom cone base (positive contribution, $L)$. The effect of the reduced number of neighbors for the atom at the cone edge could also be called the edge-tension energy and is obviously proportional to the length of the cone base. The total cone energy, $E$ is thus $E=n D+B+I+L$. Note that $B$, $I$, and $L$ depend on the size of the cone, i.e. on the total number of atoms, $N$, that make it, but in a very different functional manner. The excess energy of the cones is obviously 


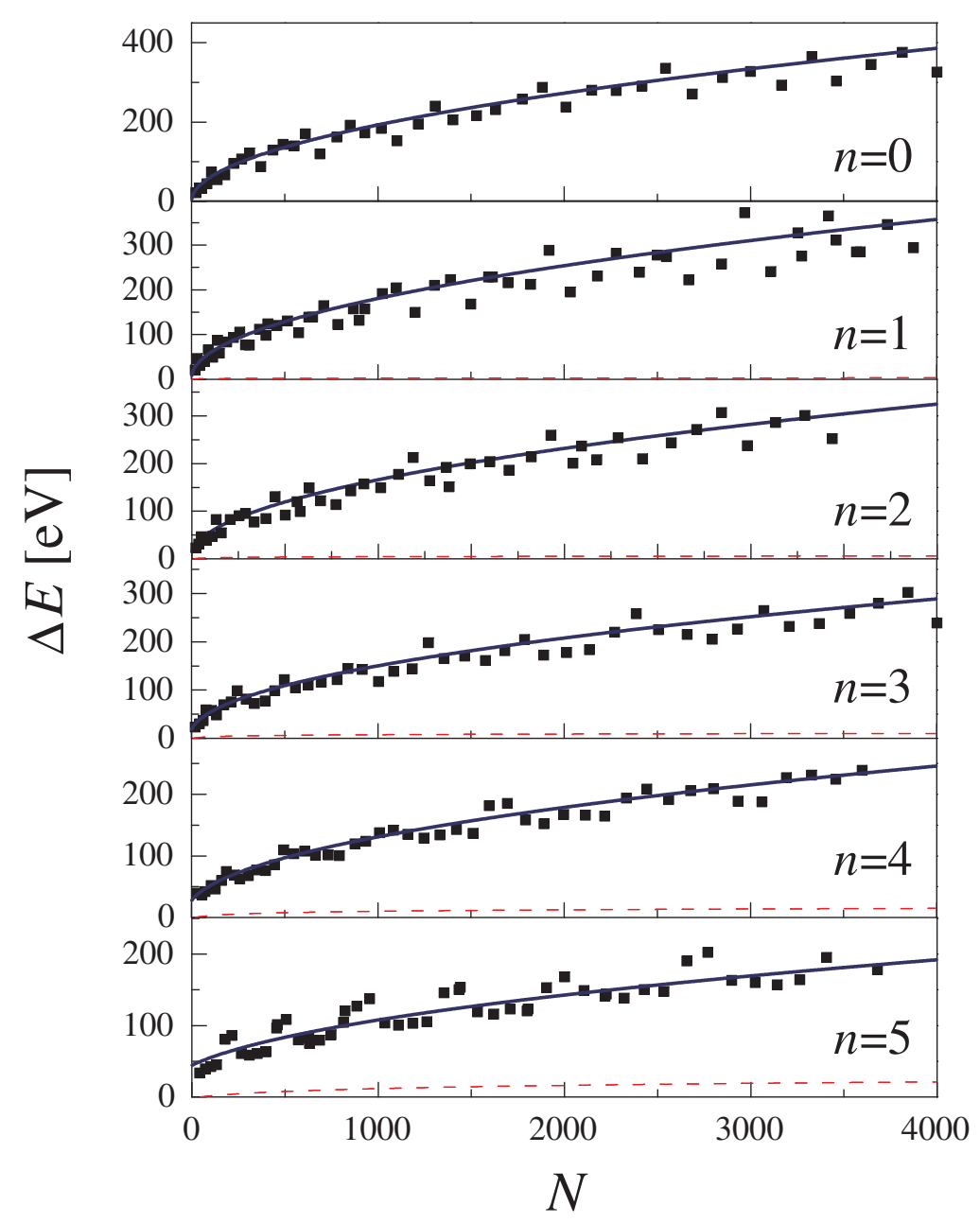

Figure 3. Energies of carbon cones as a function of the total number of carbon atoms. In addition to results for $n=1, n=2, n=3, n=4$, and $n=5$ cones, the data for " $n=0$ cones" i.e. graphene disks is also shown. The numerical results in these plots are denoted by full squares. Full thick lines are a result of analytical expression for the cone energy, Eq. (77). The thin dashed lines denote the bending (curvature) contribution to the cone energy, Eq. (2).

given by

$$
\Delta E=n D+B+L .
$$

The total area, $A$ of the conical shape can be approximately written as

$$
A=\pi \sin \left(\frac{\theta_{n}}{2}\right)\left\{r_{\text {max }}^{2}-r_{\min }^{2}\left[1-\sin \left(\frac{\theta_{n}}{2}\right)\right]\right\},
$$

so that the total number of atoms in the shape is

$$
N=A / A_{c},
$$

where the area per atom in the infinite planar sheet of graphene is given by $A_{c}=r_{0}^{2} \sqrt{3} / 2$. For the equilibrium nearest neighbor C-C distance in graphene, $r_{0}$, Brenner's potential 
predicts a value of [21] $r_{0}=1.4204 \AA$. The cone-edge energy depends on the total number of entities along the bottom basis of the cone. The total length of the bottom base edge is $2 r_{\max } \sin \left(\theta_{n} / 2\right) \pi$, so that

$$
L=2 \delta r_{\max } \pi \sin \left(\frac{\theta_{n}}{2}\right),
$$

where $\delta$ is the average line tension energy per unit length of the cone base. Combining equations (3), (21), and (6) yields the analytical expression for the dependence of the excess energy on the $r_{\max }$ parameter of the cone:

$$
\Delta E=n D+\pi c_{0} \frac{\cos ^{2} \theta_{n}}{\sin \theta_{n}} \ln \left(\frac{r_{\max }}{r_{\min }}\right)+2 \delta r_{\max } \sin \left(\frac{\theta_{n}}{2}\right) .
$$

The dependence of $r_{\max }$ on $N$ can be obtained from equations (41) and (5) which yields

$$
r_{\max }=\sqrt{\frac{N A_{c}}{\pi \sin \left(\frac{\theta_{n}}{2}\right)}+r_{\min }^{2}\left[1-\sin \left(\frac{\theta_{n}}{2}\right)\right]} .
$$

As mentioned earlier, the $r_{\text {min }}$ parameter is somewhat difficult to fix, but it can be estimated on the basis of constructions shown in Fig. 1. Values of $r_{\min }=\left\{r_{0} / \sin \left(\theta_{1} / 2\right)\right.$, $\left.r_{0} \sqrt{3} / \sin \left(\theta_{2} / 2\right), r_{0} \sqrt{3} / \sin \left(\theta_{3} / 2\right), r_{0} \sqrt{21} /\left[2 \sin \left(\theta_{4} / 2\right)\right], 2 r_{0} \sqrt{3} / \sin \left(\theta_{5} / 2\right)\right\}$ were used for $n=\{1,2,3,4,5\}$ constructions from Fig. 1, respectively. For $r_{\max } \gg r_{\min }$, the precise value of $r_{\min }$ becomes irrelevant. It can be seen in Fig. 3 that Eq. (7) (full thick lines) nicely accounts for numerical data on cone energetics for all $n$ values. In these calculations, the core disclination energy and the graphene bending rigidity were set to $D=1.83 \mathrm{eV}$ and $c_{0}=0.83 \mathrm{eV}$, respectively, in agreement with the values found in Ref. [21], and the best-fit value for $\delta$ was found to be $\delta=1.30 \mathrm{eV} / \AA$ (the same value was used for all $n$ values of the cones). This should be the edge energy lost per unit length of the edge due to the reduced coordination of the carbon atoms situated on the cone edge. The atoms at the edge can have one, two, but also three nearest neighbors (see cones in 2). The energy that is lost due to a missing neighbor should be about $2.46 \mathrm{eV}\left(\left|\epsilon_{b}\right| / 3\right)$. The exact value of $\delta$ depends on the edge of a particular cone considered, i.e. the number of singly, doubly, and triply coordinated carbon atoms and their distances. These characteristics depend both on the construction of the cone and on its total size $\left(r_{\max }\right)$. This explains the reason for scatter of the data in Fig. 3- cones of different sizes generally have differently coordinated edges, so it may happen that in a cone of a particular size all of the edge atoms are doubly or triply coordinated, which is energetically favorable case (this is the case for all cones shown in Fig. 3). Some other sizes may have edges that contain a lot of singly coordinated edge atoms. This is an interesting combinatorial problem that I shall not dwell too much upon, as the details of the shape that depend on the precise arrangement of atoms are not of interest to this article. Nevertheless, it should be noted that the value of $\delta$ obtained from the fits can be nicely explained on the basis of energetics of C-C bonding. An important objection that could be made here is that the effective Brenner's potential cannot be reliably used in situations when there are dangling bonds. In Ref. [23] it was suggested 
that Tersoff's version of carbon-carbon bonding overestimates the excess dangling bond energy. Complex non-pairwise-additive effects may be also expected in cases when there are dangling bonds and these could be beyond the reach of the potential model used in this study. Nevertheless, these effects can be expected only to renormalize the value of $\delta$, while the effective "line-tension" energy should still remain a useful concept even in that case. Even if the dangling bonds of $\mathrm{C}$ atoms at the cone edge were saturated by adsorption of hydrogen atoms [24, 25, 26], the reasoning in terms of the edge (line) tension energy would still be useful, only the value of $\delta$ would differ (see below). It is also appropriate to note here that the calculations that treated the dangling bonds in the same fashion as described above have been used in a molecular dynamics scheme to simulate the dynamics of carbon "ribbons" that may form cylinders (nanotubes) by saturating some of their dangling bonds [15].

Looking at the data in Fig. 3] it may seem that the edge tension energy dominates the cone energetics. This is evident both from the scatter of the data and from the apparent $\sqrt{N}$ behaviour of the excess energy. This is indeed the case except perhaps for $n=5$ cones, for which the bending contribution to the cone energy becomes important. This can be seen from the dashed lines in Fig. 3 that indicate the bending contribution to the cone energy [Eq. (2)]. This is in fact one of the important results of this study: the bending/curvature contribution to the cone energetics is secondary when compared to the dangling bond/edge energy, so the considerations that concentrate exclusively on this part of the cone energy in order to explain the occurrence of the cones with particular opening angle in fact deal with rather small contribution to the total cone energy [4] (at least in cases when the bonds are not saturated by hydrogen atoms, see below).

It is instructive to compare the analytic expressions for the cone energetics in a single plot, as is done in Fig. 4 (note the log scales on both axes and that the curve corresponding to graphene disks is a straight line). It can be seen that the graphene disks have the smallest excess energies for small number of $\mathrm{C}$ atoms $(N<50)$. When the number of atoms in the structures reaches about $N=50$, the excess energies of all the cones except the $n=5$ cone, become smaller than the excess energy of the disk. At this point the radius of the disk is about $0.7 \mathrm{~nm}$. When $N$ reaches about 150 atoms, the excess energy of $n=5$ cones becomes the smallest and remains so as $N$ increases. The transition region in the cone sizes that is observed in energetics of the cones smaller than about 150 atoms is a consequence of the interplay of different contributions to the excess energy of the cone. After a critical size of the graphene disk $(N \approx 50)$ the cone structures become energetically preferable, since the energy that is lost due to creation of disclinations is compensated by the reduced length of the cone edges in comparison with the length of the graphene disk. For large enough cones $(N>150)$, this effect dominates the energetics of the structures considered, and $n=5$ cones thus have the lowest excess energies, although they contain five pentagonal disclinations and their surface has the largest curvature. 


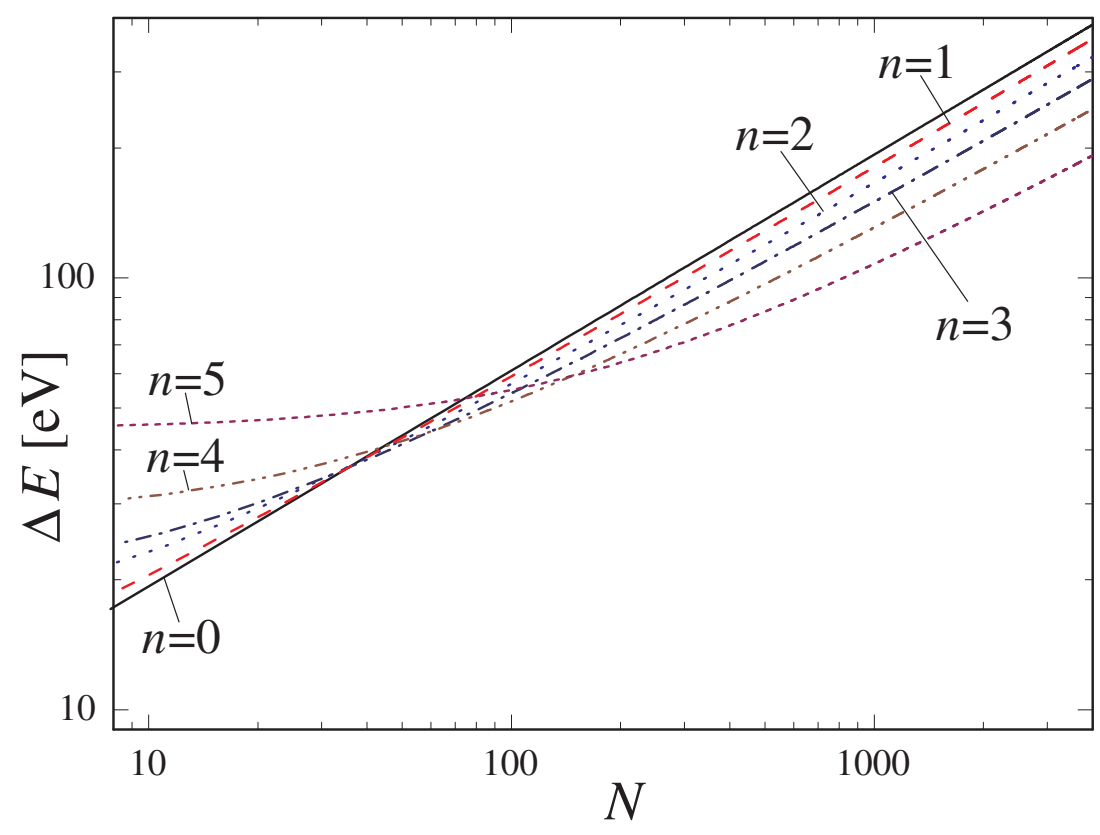

Figure 4. Analytic (continuum) expressions for the cone energetics as a function of total number of atoms in the cones for the case when the dangling carbon bonds at the cone edge are not saturated $(\delta=1.30 \mathrm{eV} / \AA)$.

It should be instructive to see how all these conclusions change if the dangling bonds were saturated by hydrogen atoms. This may be the case for carbon cones obtained by pyrolysis of hydrocarbons [6]. The energy of C-C bond in graphene is [19] $4.93 \mathrm{eV}$, while the energy of the $\mathrm{C}-\mathrm{H}$ bond is [19] $4.526 \mathrm{eV}$. Thus the energy that is lost when replacing the $\mathrm{C}-\mathrm{C}$ with $\mathrm{C}-\mathrm{H}$ bond is $0.404 \mathrm{eV}$. This is also the energy that is lost per carbon atom at the cone edge when one of its bonds has been saturated by $\mathrm{H}$ atom. This is significantly smaller from $2.46 \mathrm{eV}$ lost per carbon atom with a missing carbon neighbor. The line tension energy in this case is thus $\delta=0.404 / 2.46 \times 1.30 \mathrm{eV} / \AA=0.213 \mathrm{eV} / \AA$, more than six times smaller than in the case of unsaturated bonds. Figure 5 shows how the energetics of cones and disks whose edges are saturated by hydrogen atoms changes with the total number of carbon atoms in the structures. It can be seen that the graphene disks are the structures with the lowest excess energies up to total number of about $N=2000$ carbon atoms (compare this with $N=50$ in the unsaturated bond case). When $N>2000$, the cones have lower excess energies than graphene disks. Again, the $n=5$ cones are the most favourable in this respect. There is also an interesting crossover in cone energetics when $N<2000$ - note how the curve for $n=5$ crosses the $n=4$ and $n=3$ curves for $N \approx 170$ and $N \approx 700$, respectively. In this case, the bending contribution to the cone energy becomes important even for large cones. For example, for $n=5$ cone containing $\approx 9000$ carbon atoms, the bending contribution to its energy is $B=27.1 \mathrm{eV}$, while the edge energy is $L=39.1 \mathrm{eV}$. 


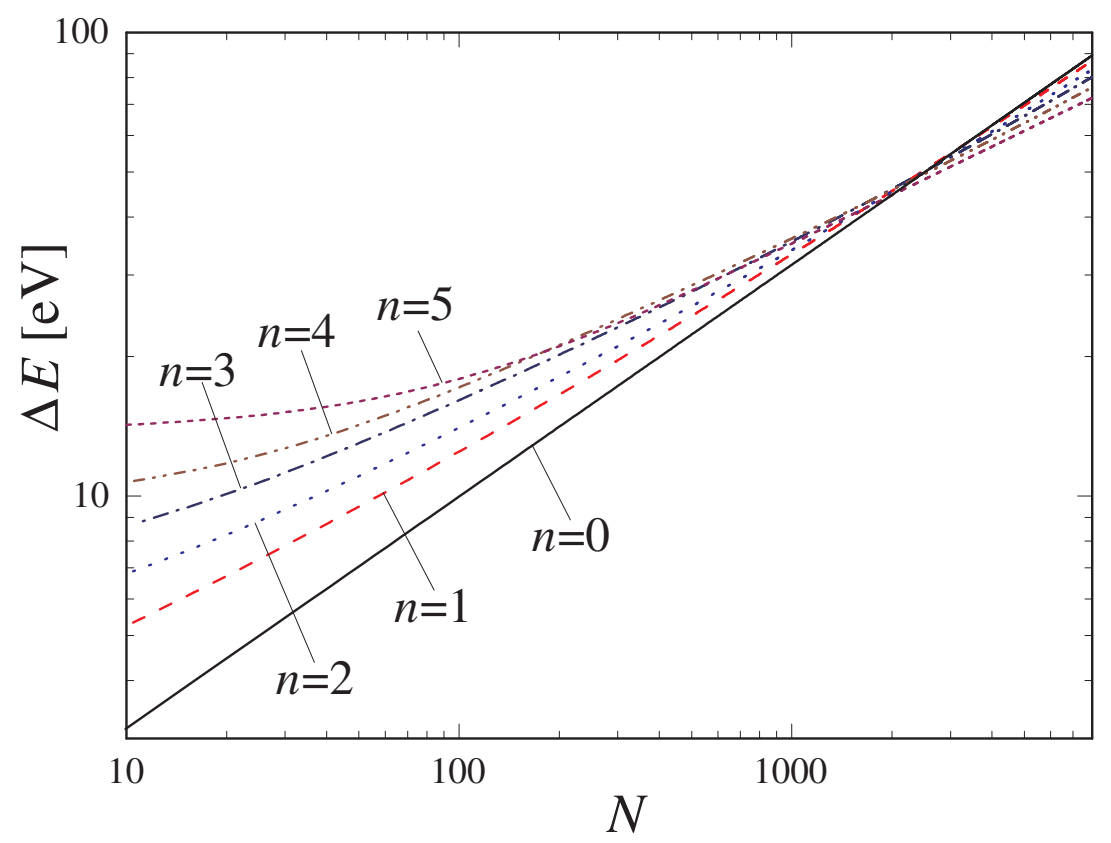

Figure 5. Analytic (continuum) expressions for the cone energetics as a function of total number of atoms in the cones for the case when the dangling carbon bonds at the cone edge are saturated with hydrogen atoms $(\delta=0.213 \mathrm{eV} / \AA)$.

Intriguingly, for the case of carbon cones whose edge atoms are saturated by hydrogen atoms, the flat disk structures have the lowest excess energies up to a much larger number of atoms than in the case of unsaturated, dangling carbon bonds at the cone edges. The crossover region at which the cones become preferred energywise is in this case positioned at significantly larger number of carbon atoms (2000 vs 150 for the dangling bonds case).

\section{Discussion}

This study has demonstrated that graphene cones are superior to graphene disks with respect to their excess energy already for structures made of relatively small number of atoms $(N>50$ for the dangling bond case and $N>2000$ for the hydrogen saturated bonds at the cone edge). For structures containing very large number of atoms, $n=5$ cones always have the smallest excess energy. For the dangling bond case, of all the shapes considered in this study that contain more than $N=150$ atoms, the $n=5$ cones have the smallest excess energy. One could speculate that this may be one of the reasons for initial experimental observation of only $n=5$ structures [4, 5. Later experiments have detected all possible cones [6] and yields of cones of different $n$-type show a nonmonotonic dependence on the number of disclinations, $n$. It would be tempting to assign this effect to the details of energetics of carbon cones in the cross-over region of sizes $(50<N<150)$. This explanation would require that the stable seeds for the cone growth are nucleated in this interesting region of sizes. Of course, one should keep in 
mind that the growth of these structures is most likely governed by kinetics and entropy in addition to energetics.

Intriguingly, it has been shown that the energetics importantly changes when one considers the cones whose edges are saturated by hydrogen atoms. In that case, the crossover region is positioned at much larger number of atoms, and the flat graphene disks are the lowest excess energy structures up to about 2000 carbon atoms. It is thus plausible that the yields of different cone structures will be different depending on whether the hydrogen atoms are present in the production process, assuming of course that they have an active role in stabilizing the carbon edges.

In summary, it has been demonstrated that the cone edge energy is the most important contribution to the total energy for cones that have more than about 150 (2000) carbon atoms. The bending energy is significantly smaller from the dangling bond energy and becomes progressively less important for larger cones, since it scales with the total number of atoms, $N$, as $\log (N)$, while the dangling bond energy scales as $\sqrt{N}$. For cones whose edges are saturated by the hydrogen atoms, the consideration of the bending contribution to the total energy is necessary for the structures containing up to about 2000 carbon atoms.

\section{Acknowledgments}

This work has been supported by the Brain Gain Programme of the National Foundation

for Science, Higher Education, and Technological Development of the Republic of Croatia under Award Number 02.03/25. and by the Ministry of Science, Education and Sports of Republic of Croatia through the Research Project No. 035-0352828-2837 ("Shapes and structures of nanoscale systems dictated by competition of energies").

[1] M. Terrones and H. Terrones, Phil. Trans. R. Soc. Lond. A 361, 2789 (2003).

[2] H.W. Kroto, J.R. Heath, S.C. O'Brien, R.F. Curl, and R.E. Smalley, Nature 318, 162 (1985).

[3] S. IIjima, Nature 354, 56 (1991).

[4] M. Ge and K. Sattler, Chem. Phys. Lett. 220, 192 (1994).

[5] K. Sattler, Carbon 33, 915 (1995).

[6] A. Krishnan, E. Dujardin, M.M.J. Treacy, J. Hugdahl, S. Lynum, and T.W. Ebbesen, Nature 388, 451 (1997).

[7] S.P. Jordan and V.H. Crespi, Phys. Rev. Lett. 93, 255504 (2004).

[8] P.E. Lammert and V.H. Crespi, Phys. Rev. B 69, 035406 (2004).

[9] J.A. Jaszczak, G.W. Robinson, S. Dimovski, Y. Gogotsi, Carbon 41, 2085 (2003).

[10] A. Šiber, Phys. Rev. E 73, 061915 (2006).

[11] B.K. Ganser, S. Li, V.Y. Klishko, J.T. Finch, and W.I. Sundquist, Science 283, 80 (1999).

[12] P.J.F. Harris, Carbon 45, 229 (2007).

[13] M.M.J. Treacy and J. Kilian, Mat. Res. Soc. Symp. Proc. 675, W2.6 (2001).

[14] S. Ihara, S. Itoh, K. Akagi, R. Tamura, and M. Tsukara, Phys. Rev. B 54, 14713 (1996).

[15] D.H. Robertson, D.W. Brenner, and C.T. White, J. Phys. Chem. 96, 6133 (1992).

[16] J. Han and R. Jaffe, J. Chem. Phys. 108, 2817 (1998).

[17] H.W. Kroto, Nature 329, 529 (1987).

[18] S. Reich, L. Li, and J. Robertson, Phys. Rev. B 72, 165423 (2005).

[19] D.W. Brener, O.A. Shenderova, J.A. Harrison, S.J. Stuart, B. Ni, and S.B. Sinnott, J. Phys.: Condens. Matter 14, 783 (2002). 
[20] W.W. Hager and H. Zhang, Siam J. Optim. 16, 170 (2005).

[21] A. Šiber, Nanotechnology 17, 3598 (2006).

[22] H.S. Seung and D.R. Nelson, Phys. Rev. A 38, 1005 (1988).

[23] V. Crespi, Phys. Rev. Lett. 82, 2908 (1999).

[24] K. Kobayashi, Phys. Rev. B 61, 8496 (2000).

[25] S. Compernolle, B. Kiran, L.F. Chibotaru, M.T. Nguyen, and A. Ceulemans, J. Chem. Phys. 121, 2326 (2004).

[26] O.A. Shenderova, B.L. Lawson, D. Areshkin, and D.W. Brenner, Nanotechnology 12, 191 (2001). 